Research article

\title{
The quantitative genetics of two life history trade-offs in the yellow dung fly in abundant and limited food environments
}

\author{
W. U. BLANCKENHORN ${ }^{1, *}$ and A. HEYLAND ${ }^{2}$ \\ ${ }^{1}$ Zoologisches Museum, Universität Zürich, Winterthurerstrasse 190, CH-8057 Zürich, Switzerland; \\ ${ }^{2}$ Department of Zoology, 223 Bartram Hall, PO Box 118525, University of Florida, Gainesville, FL \\ 32611-8525, USA \\ (*author for correspondence, fax: +41-1-635-4780; e-mail: wolfman@zoolmus.unizh.ch)
}

Received 14 May 2004; accepted 27 August 2004

Co-ordinating editor: Leimar

\begin{abstract}
The trade-offs between body size and development time and between egg size and egg number (clutch size) are central to life history theory, but evidence for them, particularly in terms of genetic correlations, is equivocal. For the yellow dung fly Scathophaga stercoraria (Diptera: Scathophagidae), we investigated variation in phenotypic and genetic variances and covariances, i.e. heritabilities and genetic correlations, of these life history traits (plus diapause) in benign and stressful larval field or adult laboratory food environments. We found both trade-offs to be weak, as evidenced by low phenotypic and genetic correlations, but stronger in the food limited environments. Broad sense heritabilities were generally significant for all traits considered, whereas the narrow sense heritabilities for egg and clutch size were nil. With regard to the question of how environmental stress affects heritabilities, we found a whole range of responses within one single species depending on the traits considered. All three possible patterns occurred, i.e. increased $h^{2}$ due to increased $V_{\mathrm{G}}$ or decreased $V_{\mathrm{P}^{\prime}}$ decreased $h^{2}$ due to increased $V_{\mathrm{P}^{\prime}}$ and no change in $h^{2}$ due to increased $V_{\mathrm{G}}$ and $V_{\mathrm{P}}$. These can be explained by the particular ecological circumstances yellow dung flies face in their natural environment. Nevertheless, the majority of patterns was consistent with the idea that stressful conditions amplify phenotypic differences between genotypes. Such variable responses of traits even within one organism underscores the complexity of this issue and may well explain the multiple patterns found in various organisms.
\end{abstract}

Key words: environmental stress, food limitation, genetic correlation, heritability, propagule size, Scathophaga stercoraria

\section{Introduction}

The evolutionary analysis of quantitative traits depends on accurate assessment of their genetic variation and covariation. The genetic variation of a trait is commonly expressed as its heritability, $h^{2}=V_{\mathrm{G}} / V_{\mathrm{P}^{\prime}}$ denoting the fraction of the phenotypic variance $V_{\mathrm{P}}$ that is genetic, whereby in the simplest case $V_{\mathrm{P}}=V_{\mathrm{G}}+V_{\mathrm{E}}$ is the sum of the genetic and environmental variance 
components (Falconer, 1989; Roff, 1997). Analogously, the phenotypic covariance between two traits is the sum of the genetic and environmental covariances, $\mathrm{COV}_{\mathrm{P}}=\mathrm{COV}_{\mathrm{G}}+\mathrm{COV}_{\mathrm{E}}$ (Roff, 1997, p. 77). Quantitative traits are typically correlated, so they do not evolve independently and may even constrain each other's evolutionary response (Price and Langen, 1992). In combination with quantitative measurements of natural and sexual selection, genetic estimates permit prediction of the evolutionary change of particular traits (Lande and Arnold, 1983; Arnold and Wade, 1984; Falconer, 1989; Roff, 1997; Lynch and Walsh, 1998). They are therefore indispensable when investigating the evolutionary consequences of ecological phenomena.

Quantitative genetic estimates are generally thought to be population- and environment-specific (Falconer, 1989, p. 164). This raises the question of how consistent heritabilities of particular traits are for a given species or population across environments. In particular, there has been much recent discussion about whether and how heritable variation should change in stressful vs. benign or field vs. laboratory environments (Hoffmann and Parsons, 1991; Roff, 1996; Weigensberg and Roff, 1996; Møller and Swaddle, 1997; Hoffmann and Merilä, 1999). This is important because the inconsistency of and uncertainty about genetic estimates severely limits inferences drawn about trait evolution in variable environments. This curtails their accessibility for many behavioural and evolutionary ecologists, which is clearly undesirable. Hoffmann and Merilä (1999) have recently reviewed the evidence for effects of unfavourable conditions on genetic trait (co)variation. They list a total of eight genetic mechanisms proposed to explain such effects. These fall into the three possible categories predicting increased, decreased or no change in heritabilities (or make no clear prediction). Hypothesis 1: Stressful conditions can increase genetic (co)variation i.e., decrease $V_{G}$ and thus $h^{2}$, if they amplify phenotypic differences between genotypes (Hoffmann and Parsons, 1991). Hypothesis 2: Conversely, stressful conditions can (a) increase environmental variation i.e., increase $V P_{G}$ including variation due to common environment (in the denominator), or (b) lead to the genetic variation (in the numerator) of organisms not being fully expressed (i.e., decrease $V_{G}$ Gebhardt-Henrich and van Noordwijk, 1991), both resulting in lower $h^{2}$ or genetic correlations. Hypothesis 3: Finally, if both genetic and environmental (i.e. phenotypic) variation are increased, no change in $h^{2}$ may result (e.g. Blanckenhorn, 2002). Hoffmann and Merilä (1999) concluded that "recent studies suggest frequent changes in additive genetic variance, but no consistent effects of unfavourable conditions on the heritability of traits" ( $p$. 100). They called for more work addressing specific hypotheses.

Hypothesis 1 above predicts that presumed trade-offs underlying the evolution of adaptive life histories are likely to be detected only in resource limited, i.e. stressful environments, because when resources are not limited, individuals 
can invest maximally in all traits. Or put more simply in another way: good environments, or good condition, often mask expected life history costs and trade-offs (Ojanen et al., 1979; van Noordwijk and de Jong, 1986; Alatalo et al., 1990; Schluter et al., 1991; Rowe and Houle, 1996). Roff (1992, p. 356) remarked that most investigations of trade-offs are weak because they are correlational and unmanipulated. Food limitation is one of the most common environmental stresses in natural populations. To date, a handful of studies have specifically studied effects of nutrition on genetic variation primarily of body size, and these have yielded variable results with regard to the hypotheses above (Ebert et al., 1993; de Moed et al., 1997; Grill et al., 1997; Lazarevic et al., 1998; summarized in Hoffmann and Merilä, 1999; but also compare Hoffmann and Parsons, 1991; Simons and Roff, 1994; Roff, 1996; Weigensberg and Roff, 1996; Merilä, 1997; Møller and Swaddle, 1997; Blanckenhorn, 2002). In this study, we investigate variation in phenotypic and genetic variances and covariances, i.e. heritabilities and genetic correlations, of key life history traits in benign and stressful larval or adult food environments for the yellow dung fly, Scathophaga stercoraria (Diptera: Scathophagidae).

In one field experiment, we investigated effects of varying larval nutrition on genetic variance in body size (hind tibia length, HTL), development time, and diapause induction, three important fitness components in the yellow dung fly (Blanckenhorn, 1998; Jann et al., 2000) and in general. We especially focussed on covariation between the first two traits. Diapause was analyzed primarily because this naturally occurring phenomenon split our data set into two parts. A positive correlation between body size and development time (i.e. a trade-off between the two) is generally expected and features prominently in many life history models, as it takes time to grow bigger (Roff, 1992, 2000; Kozlowski, 1992; Stearns, 1992; Abrams et al., 1996). However, often enough positive correlations are not found, some studies even yielding negative correlations (e.g. Klingenberg and Spence, 1997; Kause et al., 1999; reviewed in Roff, 2000). Aspects of this study were already published in another context (Blanckenhorn, 1998), but genetic estimates in the various larval food environments were not reported.

In a second, laboratory experiment, we analogously investigated effects of limited adult prey availability on two key female fitness components, egg size and egg number (i.e. clutch size). A trade-off, i.e. a negative correlation between these two traits is central to life history theory and often expected because a given amount of energy can be invested either in few large or many small propagules (Smith and Fretwell, 1974). Negative phenotypic correlations have been reported in some organisms, but these show inconsistent variation among taxonomic groups and environments, and often enough positive relationships appear (reviewed in Roff, 1992, p. 357; Stearns, 1992, p. 218). So evidence for this trade-off is also equivocal. In particular, even though both 
propagule size (Roff, 1992, p. 359; 1997, p. 261) and clutch size (Roff, 1992, pp. 322 and 360) have been shown to be heritable in a variety of species, evidence for a negative genetic correlation, which is expected if the trade-off is manifested in evolutionary terms, is scarce, particularly in insects (Roff, 1992, p. 358). Snyder (1991), Ebert (1993), Sinervo and Doughty (1996) and Mappes and Koskela (2004) report negative genetic correlations in sticklebacks, water fleas, lizards and voles, respectively, while Lessells et al. (1989) and $\mathrm{Su}$ et al. (1997) report no or even positive genetic correlations in snow geese and rainbow trout. For insects, Schwarzkopf et al. (1999) and Czesak and Fox (2003) demonstrated a negative correlated genetic response of fecundity to selection on egg size in Drosophila melanogaster and a seed beetle, respectively. By presenting food environment-specific estimates of phenotypic and genetic variation and covariation in these two pairs of life history traits, body size and development time on the one hand, and egg size and clutch size (egg number) on the other hand, we specifically address the hypotheses listed above on the relationship of genetic (co)variation to environmental stress (Hoffmann and Merilä, 1999).

\section{Methods}

Larval food limitation: body size, development time and diapause

For 12 successive weeks (henceforth cohorts) starting 9 September 1995 (Blanckenhorn, 1998), field-collected S. stercoraria females were allowed to copulate twice with the male they were caught with and lay eggs on a portion of dung in the laboratory soon after capture. To establish genotype-by-larval environment interactions, each female's clutch was split among three increasingly stressful larval food environments of overabundant $(20 \mathrm{~g})$, somewhat limiting (10 g) and severely limiting (5 g) amounts of defrosted, uniform cow dung (cf. Amano, 1983). (These sibships may have included a proportion of half-sibs because females likely had stored sperm from previous copulations. As the last male to mate fertilizes about $80 \%$ of the eggs (Parker, 1970) and each pair was allowed to copulate twice before laying, the probability that subsequent offspring were full- rather than half-sibs was about 0.96. Should half-sibs have been included in the sample, this would in general have increased the within-family (i.e. error) variance and is hence conservative.) There were eight families of 10 larvae per cohort and environment, each in a plastic container capped with a toilet paper lid so that emergence and egg-to-adult development times could be scored, and the hind tibia length (HTL) of each individual measured as an index of body size. Containers were kept for a maximum of $24 \mathrm{~h}$ at room temperature, so hatching of the larvae could be 
scored. They were then placed on the ground in the open under the roof of a shed (yielding permanent shade). Developing individuals were thus exposed to seasonal changes in environmental conditions (notably temperature, photoperiod and humidity). We checked for adults emerging prior to winter, as well as after winter, at least every other day. Only the cohorts 2-6 featuring substantial numbers of flies emerging prior to winter were analyzed (cohort 1 flies almost all died; see Blanckenhorn, 1998). More detail on these methods is given in Blanckenhorn (1998). Here we only consider variation among larval environments, averaging over all cohorts by statistically removing this variation as a blocking factor in the ANOVA.

Adult prey limitation: egg and clutch size

To assess the relationship between egg volume and clutch size in the laboratory at limited and unlimited adult food, we used a standard mother/daughter, halfsib, split-brood (two container) quantitative genetic design (Roff, 1997). Contrary to the larval experiment, the experiments at unlimited and limited food were performed at different times (summer and winter 1998, respectively), so no genotype-by-adult food environment interactions could be computed. The data sets could nevertheless be compared because they were performed at identical environmental conditions. To minimise carry-over maternal effects at either food treatment, the parental generation was the laboratory F2 (and their offspring generation the F3) derived from females originally caught at our field site in Fehraltorf near Zürich.

We used standard rearing and holding methods described in more detail in Blanckenhorn (1997). Parent and offspring adult flies were held singly in $100 \mathrm{ml}$ bottles in climate chambers at $19{ }^{\circ} \mathrm{C}, 60 \%$ r.h. and $13 \mathrm{~h}$ photoperiod. They were supplied with ad libitum sugar and water, and either ad libitum Drosophila melanogaster as prey in the unlimited food treatment, or 10 D.melanogaster per week in the limited food treatment (cf. Jann and Ward, 1999). Adult yellow dung flies can subsist on sugar and water but require prey to produce eggs and sperm (Foster, 1967; Gibbons, 1980), and the amount of prey directly affects clutch size and number, oviposition rate and egg volume (Jann and Ward, 1999). These laboratory conditions are well within the range the flies experience in their natural environment (Blanckenhorn, 1997, 1998).

Females of the F2 laboratory generation were allowed to copulate with a randomly picked male (but not her brother) from the same population 14 days after adult emergence and allowed to lay eggs on a smear of dung. In case no eggs were produced, this procedure was repeated (using the same male) after a few days until the first clutch was laid. Three of these mothers were mated with the same male (i.e. there were three dams per sire). For every mother, we counted the number of eggs laid (first clutch size) and measured the length and 
width of five randomly picked eggs from her first clutch using a binocular microscope at $40 \times$ magnification. The volume of the ellipsoid eggs was then estimated as $(4 / 3) \cdot \pi \cdot($ length $/ 2) \cdot(\text { width } / 2)^{2}$ and the mean computed. To control for common larval environment (Roff, 1997), a mother's clutch was split and 10-15 eggs were allowed to develop in each of two $50 \mathrm{ml}$ plastic containers with an overabundant amount of 20-40 g defrosted, uniform dung at climatic conditions identical to the holding conditions given above. Amano (1983) showed that at more than $2 \mathrm{~g}$ dung per larva competition has no noticeable effect on larval mortality or adult body size (cf. above). Adult body size variation was thus minimized in our study by using constant temperatures (Blanckenhorn, 1997) and abundant larval food. Three female (and one male) offspring were randomly chosen upon emergence from each of the two containers per family. They were held exactly as their parents and mated with a randomly picked male, yielding a maximum of six first clutch sizes and mean egg volumes per offspring family. The HTL of all individuals was measured after death as an index of body size.

\section{Statistical analyses}

Heritabilities $h^{2}$ of HTL, development time and diapause (a dichotomous threshold trait; Roff, 1997) in the larval experiment were calculated using standard full-sib ANOVA, and $h^{2}$ of mean egg volume and clutch size in the adult experiment were calculated using half-sib ANOVA or mean offspring-onparent regression. Their corresponding approximate standard errors were calculated as in Becker (1992) and Roff (1997), and the corresponding variance components were estimated using restricted maximum likelihood in SPSS, version 10. In the larval experiment, males and females were analysed separately because they differ in body size and development time (but not for diapause; Roff, 1997). In the adult experiment, the repeatability $R$ of egg volume within a clutch was additionally calculated (Becker, 1992). All significances stem directly from the regressions and ANOVAs. Genetic covariances between traits within individuals were computed using ANCOVAs analogous to those for $h^{2}$ (Becker, 1992; Roff, 1997). Genetic correlations $r_{\mathrm{g}}$ were calculated as the Pearson product-moment correlation among the maternal and/or paternal trait family means in the offspring generation, which are good approximations of genetic correlations resulting from ANCOVA but much more straightforward to compute (Via, 1984; Roff, 1997). The corresponding phenotypic correlations $r_{\mathrm{p}}$ were also calculated.

In the larval experiment broods were split among environments, permitting direct establishment of genotype-by-environment interactions by means of mixed model ANOVA implying variation in $h^{2}$ among environments (Fry, 1992). Variation in the phenotypic or genetic correlations between body size 
(dependent variable) and development time (independent variable) was tested via the development time by larval environment interaction term in ANCOVAs using the raw data or family means, respectively. In the adult experiment, we could not establish genotype-by-adult food environment interactions because broods were not split among environments (see above). Nevertheless, we could still analogously test for variation in the genetic parameters among environments as above.

Being a ratio, $h^{2}=V_{\mathrm{G}} / V_{\mathrm{P}}$ can increase because the numerator $\left(V_{\mathrm{G}}\right)$ increases and/or the denominator $\left(V_{\mathrm{P}}\right)$ decreases. Analogously, a correlation between two variables, $r=\operatorname{COV}(X, Y) /(\operatorname{SD}(X) \operatorname{SD}(Y))$, increases when the covariance (in the numerator) increases and/or the variances (in the denominator) decrease. Therefore, to evaluate the effect of the environment on heritabilities and genetic correlations, the variance (for $h^{2}$ ) and covariance components (for $r$ ) need to be compared in relation to the phenotypic variances. We did this using simple variance ratio $F$-Max tests.

\section{Results}

Larval food limitation: body size, development time and diapause

A total of 813 flies of 27-40 families emerged from cohorts 2-6, either before winter or after winter diapause (Table 1). Broad sense heritabilities of body size (hind tibia length, HTL) and development time increased as larvae were more food limited (Table 1). This is indicated by significant family by larval environment interactions for males (HTL: $F_{50,260}=5.67$; development time: $F_{50,260}=2.28$ ) and females (HTL: $F_{61,343}=2.10$; development time: $F_{61,343}=2.05$; all $\left.p<0.001\right)$, indicating this phenotypic plasticity has a genetic component. However, for HTL this occurred because genetic variance increased while environmental (error) variance remained stable ( $F$-Max test for male and female genetic components $(5 \mathrm{~g}$ vs. $10 \mathrm{~g}): F_{27,37}=2.13$ and 2.62 ; both $p<0.05$; environmental components: $F_{33,38}=1.19$ and 1.15 ; both $>$ ns), whereas for development time this occurred because environmental variance decreased while genetic variance remained roughly stable (male and female genetic components: $F_{27,37}=1.39$ and 1.27 ; both ns; environmental components: $F_{33,38}=4.11$ and 2.85 ; both $p<0.01$; Table 1 ). In contrast, the heritability of diapause decreased as the larval environment became more limited (i.e. stressful), and this occurred because the environmental (error) variance component increased (genetic component: $F_{40,40}=1.09$, ns; environmental component: $F_{40,40}=1.35(5 \mathrm{~g}$ vs. $10 \mathrm{~g})$ and $1.92(5 \mathrm{~g}$ vs. $20 \mathrm{~g})$; $\mathrm{ns}$ and $p<0.05$, respectively; Table 2). Note that all broad sense estimates are high and sometimes greater than one because they contain substantial amounts of 


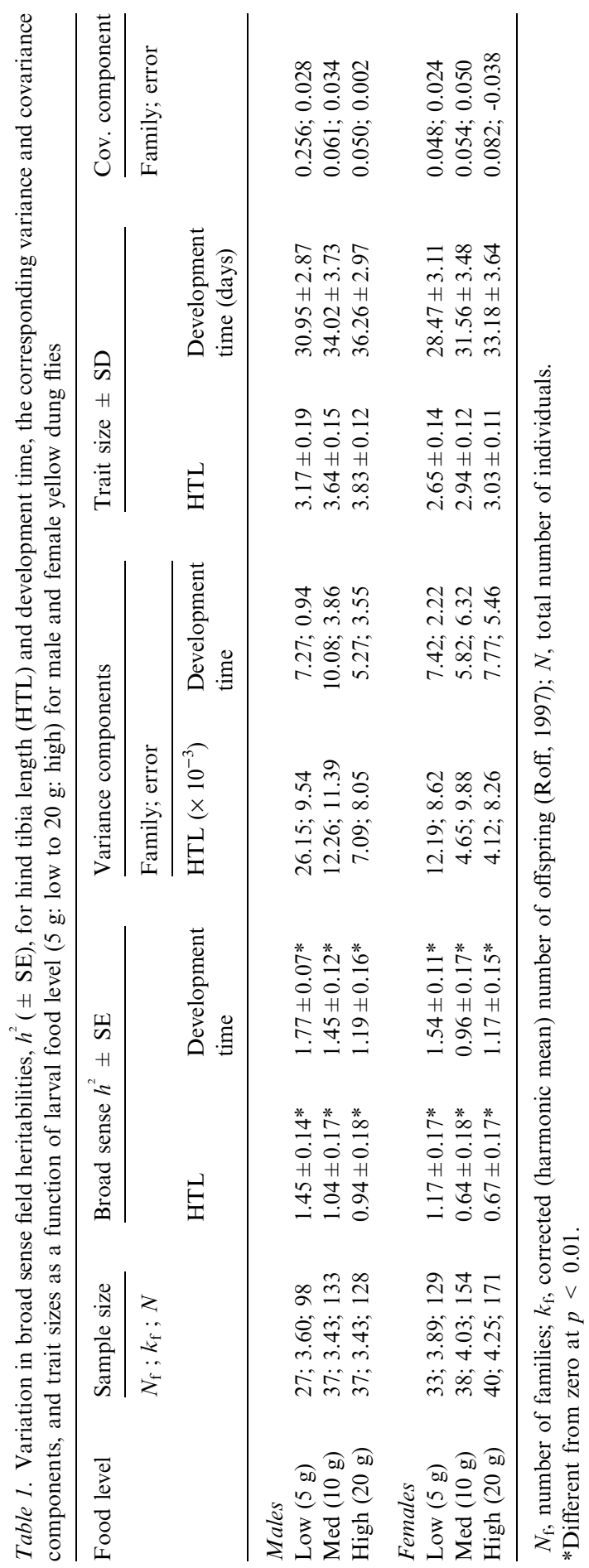


Table 2. Variation in broad sense field heritabilities $h^{2}( \pm \mathrm{SE})$ for diapause (a dichotomous threshold trait) and the corresponding variance components as a function of larval food level (5 g: low to $20 \mathrm{~g}$ : high) in yellow dung flies

\begin{tabular}{llll}
\hline Food limitation & $\begin{array}{l}\text { Sample size } \\
N_{\mathrm{f}} ; k_{\mathrm{f}} ; p\end{array}$ & $\begin{array}{l}\text { Broad sense } \\
h^{2} \pm \mathrm{SE}\end{array}$ & $\begin{array}{l}\text { Variance components } \\
\text { Family; error }\end{array}$ \\
\hline Low $(5 \mathrm{~g})$ & $40 ; 8.29 ; 0.27$ & $0.43 \pm 0.17^{*}$ & $1.27 ; 9.40$ \\
Med $(10 \mathrm{~g})$ & $40 ; 9.42 ; 0.19$ & $0.59 \pm 0.20^{*}$ & $1.17 ; 6.97$ \\
High $(20 \mathrm{~g})$ & $40 ; 9.15 ; 0.09$ & $1.26 \pm 0.34^{*}$ & $1.31 ; 4.90$ \\
\hline
\end{tabular}

$N_{\mathrm{f}}$, number of families; $k_{\mathrm{f}}$, corrected (harmonic mean) number of offspring; $p$, mean proportion diapausing (Roff, 1997). *Different from zero at $p<0.05$.

dominance, epistatic and common environment variance as well as maternal effects (Blanckenhorn, 2002).

Phenotypic and genetic (full-sib family mean) correlations between HTL and development time were of similar magnitude (Fig. 1). The strength of this relationship (and thus $r_{\mathrm{p}}$ ) increased as the larvae were more food limited, as indicated by significant development time by treatment interactions (males: $F_{2,354}=5.76, p=0.003$; females: $F_{2,448}=4.57, p=0.011$ ). The same qualitative trend was apparent for the genetic correlations $r_{\mathrm{g}}$, at least for males, but this was not significant (development time by larval treatment interactions on family means for males: $F_{2,82}=2.01$; females: $F_{2,93}=0.09$; both ns). Note that, at least in males, the correlation in the most limited food environment (5 g) was highest because the covariance (in the numerator) was higher while the overall phenotypic variances of both traits (in the denominator) remained roughly the same across all larval treatments (Table 1).

\section{Adult prey limitation: egg and clutch size}

There were 33 maternal (and 11 paternal) families with 220 female offspring at unlimited food, and 48 maternal (and 16 paternal) families with 222 female offspring at limited food. Mean offspring egg volumes and clutch sizes were greater $\left(t_{218}=23.72\right.$ and 23.99 , respectively; both $\left.p<0.001\right)$ and their phenotypic variances smaller ( $F$-Max test: $F_{221,197}=1.53$ and 2.69; both $p<0.01$ ) at unlimited food (Table 3$)$. The repeatability $( \pm \mathrm{SE})$ of egg volume within clutches was $R=0.83 \pm 0.06$ at limited food and $0.89 \pm 0.05$ at unlimited food (difference ns).

Broad sense $h^{2}$ of clutch size and egg volume were largely significant, while narrow sense $h^{2}$ were never significant, and mother/daughter $h^{2}$ were only significant at unlimited food (Table 3 ). In addition to additive genetic variance, broad sense $h^{2}$ estimates contain dominance and epistatic variance and maternal effects (note that common environment effects were excluded here), while mother/daughter estimates may still contain maternal effects, which are 


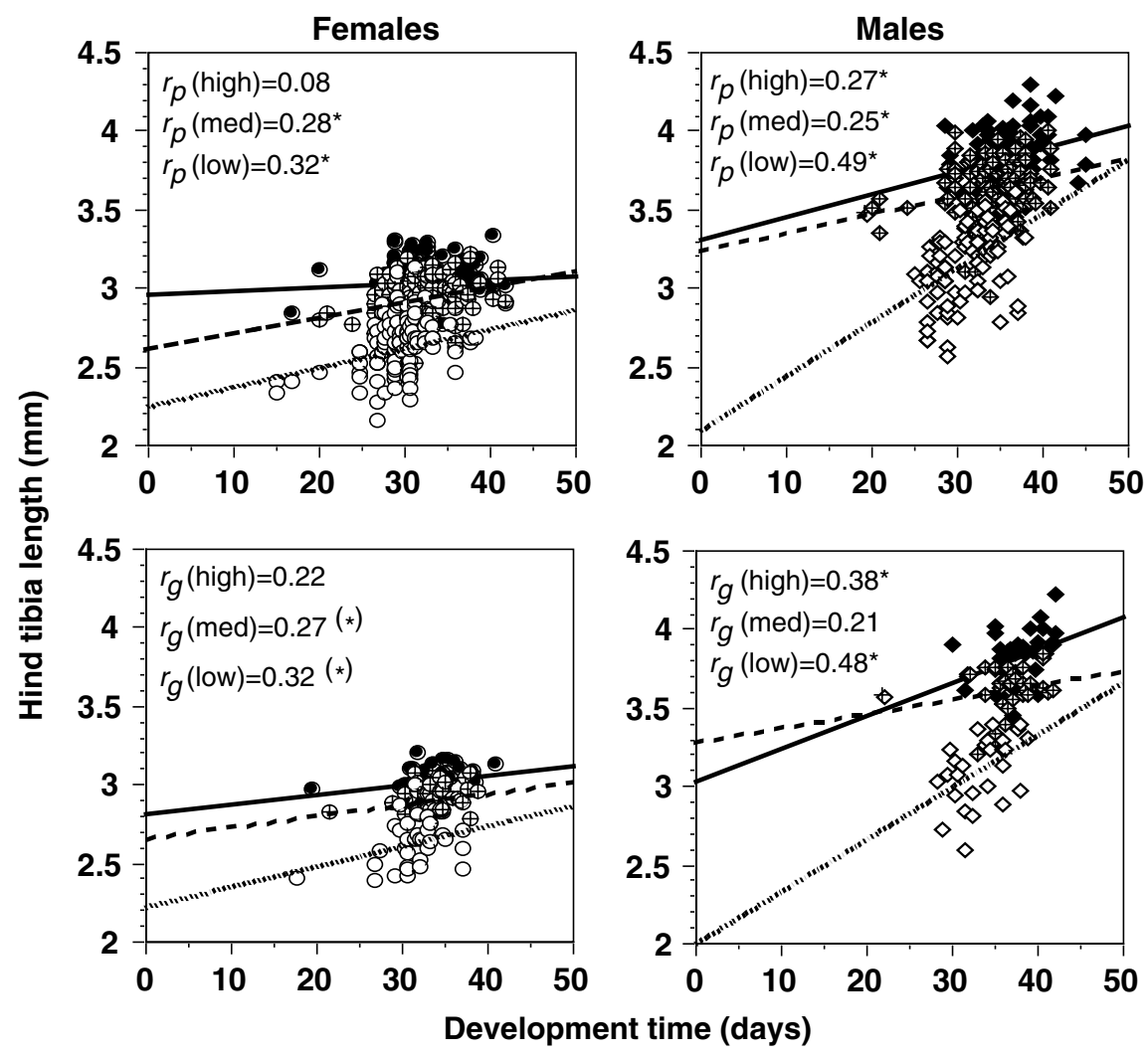

Figure 1. Phenotypic (top) and full sib family mean genetic (bottom) correlations between development time and body size at three different larval food levels (low: open symbols and dotted line; medium: grey symbols and hatched line; high: black symbols and unbroken line) for female (left) and male (right) yellow dung flies in the field (regression lines drawn beyond the extent of data so they are visible; ${ }^{*} p<0.05{ }^{(*)} p<0.10$ ).

expected to be substantial for these two reproductive traits (Falconer, 1989; Roff, 1997). For clutch size, the broad sense $h^{2}$ was lower at limited food (significant food treatment by dam interaction: $F_{21,27}=2.43, p=0.017$ ), due to an increased environmental (i.e. phenotypic; in the denominator) but at the same time stable genetic (dam) variance component (in the numerator: $F$-Max test: $F_{32,47}=3.88$ and $1.06 ; p<0.001$ and ns, respectively). For egg volume, both heritabilities were similar (food treatment by dam interaction: $F_{21,27}=1.10, p=0.340$ ), because the environmental and genetic (dam) variance components increased in conjunction at limited food ( $F$-Max test: $F_{32,47}=1.59$ and 1.74 , respectively; $p=0.073$ and 0.041 ). Box (i.e. common larval environment) effects were always ns but nonetheless removed statistically in all analyses (Roff ,1997). 


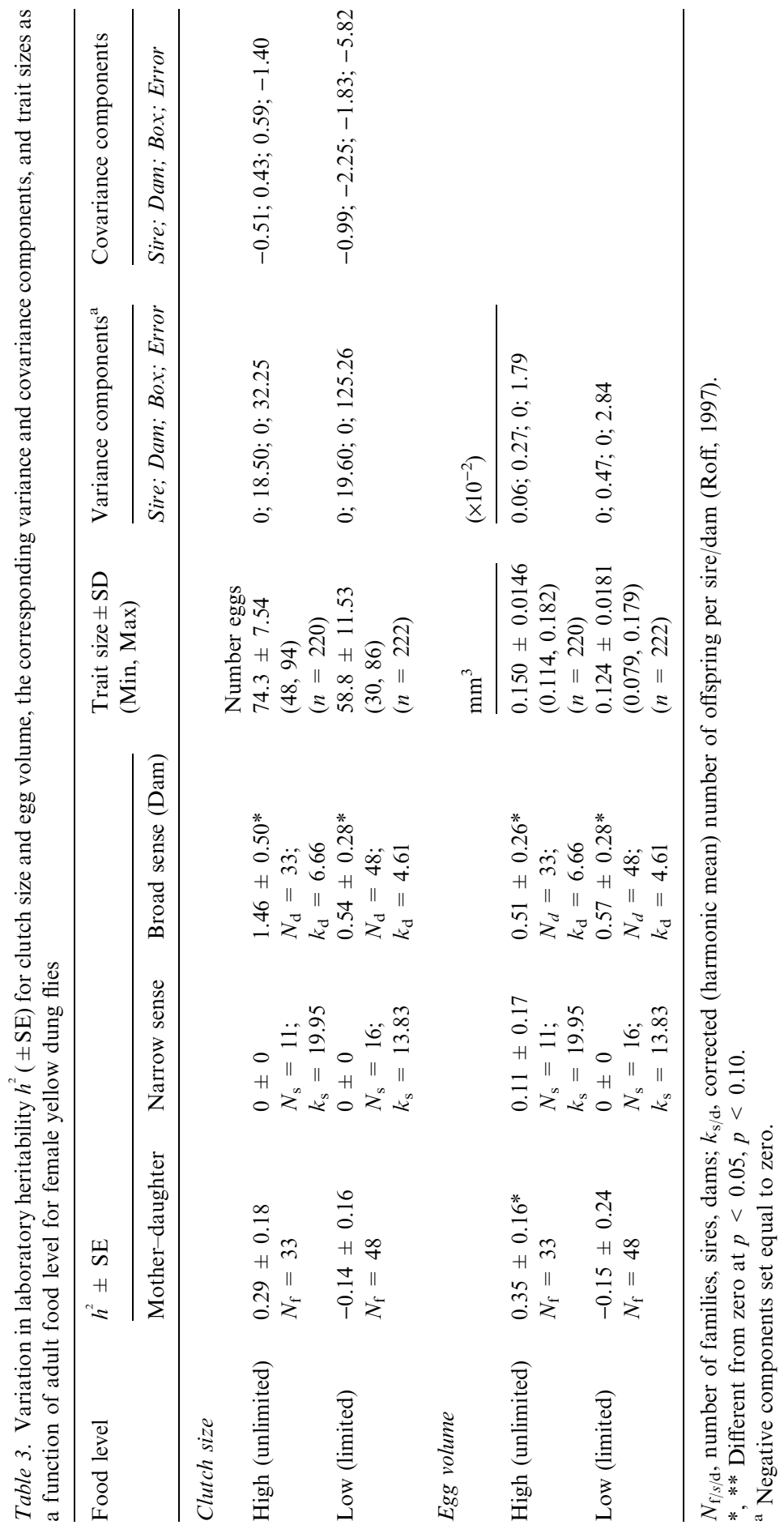


As in the larval experiment, phenotypic and genetic correlations between egg and clutch size were similar (Fig. 2). There was a slight negative phenotypic relationship (i.e. a trade-off) at limited but not at unlimited food (significant egg volume by treatment interaction with clutch size as the dependent variable: $\left.F_{1,438}=4.76, p=0.030\right)$. The analogous test was not significant for the genetic (dam or sire) correlations, at least in part due to lower power $\left(F_{1,77}=2.60\right.$ and $F_{1,23}=0.66$, respectively; both ns; Fig. 2). The dam covariance component (in the numerator) changed sign to become negative at limited food, but the phenotypic variance (in the denominator) of both traits also increased (Table 3). Furthermore, clutch size was correlated with body size at limited $\left(r_{\mathrm{p}}=0.14, p<0.05\right)$ and unlimited food $\left(r_{\mathrm{p}}=0.45, p<0.001\right)$, but egg volume was not (limited food: $r_{\mathrm{p}}=0.01$; unlimited food: $r_{\mathrm{p}}=-0.02$; both ns).

\section{Discussion}

The often assumed trade-off between body size (here hind tibia length: HTL) and development time (Roff, 1992, 2000; Kozlowski, 1992; Stearns, 1992) was present but only weak in the yellow dung fly, as phenotypic and (broad sense) genetic correlations between the two variables were low. Similarly, the classic trade-off between egg and clutch size (Smith and Fretwell, 1974) was even weaker and not significant when considering (narrow and broad sense) genetic correlations. In both cases, the correlations tended to be stronger in the more stressful, food limited environment. Broad sense (full-sib) heritabilities $\left(h^{2}\right)$ were high and generally significant for all traits considered, whereas the narrow sense (half-sib) $h^{2}$ for egg and clutch size were nil. Mother/daughter $h^{2}$ for egg and clutch size were significant only at unlimited food, probably because the
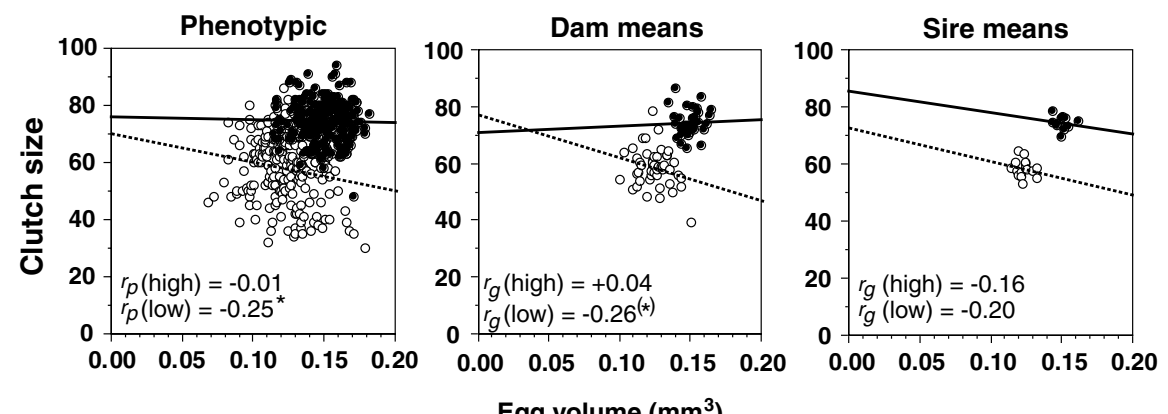

Egg volume $\left(\mathrm{mm}^{3}\right)$

Figure 2. Phenotypic, full sib (dam) and half sib (sire) family mean genetic correlations between egg volume and clutch size at two different adult food levels (low: open symbols and dotted line; high: black symbols and unbroken line) for yellow dung flies in the laboratory (regression lines drawn beyond the extent of data so they are visible; ${ }^{*} p<0.05$; $^{(*)} p<0.10$ ). 
mothers were not food limited and therefore mother and offspring environments differed for the limited food treatment (cf. Falconer, 1989). The effect of environmental stress on genetic variation, and thus the broad sense heritabilities, depended on the trait considered but showed some interesting patterns primarily consistent with the idea that stressful conditions amplify phenotypic differences between genotypes (Hoffmann and Parsons, 1991; Hoffmann and Merilä, 1999). Below we discuss these findings in more detail.

The trade-off between development time and body size is only weakly present in the yellow dung fly. This is not uncommon, and sometimes even negative correlations are found (Roff, 2000). Yellow dung fly larvae live in ephemeral habitats (dung pats) in which the degree of inter- or intra-specific competition is unpredictable in time and space, and the same probably holds true for adult prey availability in nature, if only due to variable weather conditions (cf. Blanckenhorn, 1997). In response, yellow dung flies show extensive adaptive phenotypic plasticity in growth, development and body size, emerging smaller but earlier when the dung dries up (Blanckenhorn, 1998, 1999). Adaptive variation in growth rate requires that the correlation between body size and development time be low, i.e. genetically unconstrained (Abrams et al., 1996; Blanckenhorn, 1998), as in the simplest case growth rate is calculated as size accumulated per unit development time. This was the case for both phenotypic and genetic correlations here, which were quite similar (cf. Cheverud, 1988; Roff, 1995, 1996, 1997). Unfortunately, as yet we do not know anything about the proximate regulation of this growth plasticity (cf. Kause et al., 1999; Zera and Harshman, 2001; Davidowitz et al., 2004). All larvae appear to grow at roughly the same rate (Blanckenhorn, 1999, and unpublished data) but flexibly cease growth in response to food limitation, probably mediated by changes in the critical weight inducing pupation (Davidowitz et al., 2004).

The broad sense heritabilities for diapause, HTL and development time were all very high. In this phenotypically plastic species, large common environment, dominance, epistatic and, for these traits probably to a lesser extent, maternal effect components are typical (Blanckenhorn, 2002; cf. Crnokrak and Roff, 1995). Nevertheless, we found some interesting patterns regarding the effect of larval food limitation (or stress) on these life history traits, which should not be grossly confounded by non-additive variance. For both HTL and development time heritabilities increased with larval food stress, but for different reasons. For HTL this occurred because genetic variance increased while environmental variance remained stable, whereas for development time this occurred because environmental variance decreased while genetic variance remained stable. When dung becomes limited, all flies, regardless of their "target", "genetic" size, abbreviate their development and emerge almost simultaneously, thus reducing environmental (phenotypic) variation in development time to a 
minimum. Flies also emerge much smaller. However, phenotypic variation in HTL is reduced to a lesser extent because of extensive variation in growth rates among genotypes, some (but not others) of which consequently emerge larger after the same development time (Blanckenhorn, 1998). For both HTL and development time this results in the genetic variance (in the numerator) being relatively greater in the stressful environment, resulting in higher $h^{2}$. At least in males, the covariance component similarly increased in the limited larval environment, together with the reduced phenotypic variance resulting in stronger correlations between HTL and development time. These results are thus consistent with the idea that stressful conditions amplify phenotypic differences between genotypes, thus increasing heritabilities and genetic correlations (Hoffmann and Parsons, 1991; Hoffmann and Merilä, 1999; Hypothesis 1 in the Introduction). Winter diapause induction, however, a third, threshold life history trait, showed the opposite pattern: its heritability decreased as the larval environment became more limited, as the environmental variance component increased while the genetic component remained stable, thus supporting Hypothesis 2 in the Introduction. This probably occurred because a greater proportion of flies in the food limited larval environment opted to enter diapause, thus presumably escaping some additional costs of direct development that would have increased mortality (see Fig. 1 in Blanckenhorn, 1998). The different responses of the three life history traits examined to larval food stress are thus explainable by the particular ecological circumstances yellow dung flies face in their natural environment.

Based on phenotypic and broad sense genetic data, we found merely a hint of a trade-off between egg size and egg number (clutch size) in the yellow dung fly, and only when adult food was limited. This agrees with Hypothesis 1 and the view that good nutrition (i.e. good condition) often masks expected trade-offs (Ojanen et al., 1979; van Noordwijk and de Jong, 1986; Alatalo et al., 1990; Schluter et al., 1991; Rowe and Houle, 1996), as was the case to some degree for body size and development time as well (see above). Thus resource limitation resulted in lower means but higher phenotypic variances for both clutch and egg size. A reduction in mean under food stress is perhaps not surprising. Food-stressed females, whose maximal clutch size is presumably determined at emergence (Bennettova and Fraenkel, 1981; see below), probably resorb some of their eggs and re-invest the energy gained in others. The corresponding increase in phenotypic variance indicates that food limitation increased environmental variation and/or amplified (heritable) variation among females in their ability to convert energy into eggs: when food is abundant, even females that are inefficient at converting energy into eggs can ultimately have a high reproductive output. This phenomenon thus could and should in a next step be further tracked back to the underlying physiological processes of energy conversion and allocation (cf. Kause et al., 1999; Zera and Harshman, 2001). For 
clutch size, only the environmental variance increased, whereas for egg size both genetic and environmental variance increased in conjunction (Table 3). As a result, our broad sense heritability of clutch size decreased in the food limited environment (consistent with Hypotheses 2 above), whereas it remained stable for egg volume (consistent with both Hypotheses 1 and 2, and consequently Hypothesis 3). The results for clutch size (but not necessarily egg volume) thus agree with the expectation that heritabilities in field situations, where environmental variation is typically high, should be lower than those estimated in the laboratory (Ebert, 1993; Ebert et al., 1993; Simons and Roff, 1994; Weigensberg and Roff, 1996). Our broad sense heritability estimates for egg and clutch size are roughly in line with averages reported for life history traits of other organisms (Mousseau and Roff, 1987). However, we found no narrow sense (i.e. paternal half-sib) heritability for either trait, perhaps because in yellow dung flies these traits are largely determined by the mother (see below).

In principle, the egg size/egg number trade-off can arise because of space (i.e. size) or energy limitations (Roff 1992, p. 356; Zera and Harshman, 2001). If it is primarily mediated by energy limitation of the mother, propagule size and number should be traded off if resources are limited but not necessarily if they are unlimited. This is because when resources are superabundant, the maximal number of eggs of the maximal size possible (fitting in the available space) should always be produced. Bennettova and Fraenkel (1981) have shown that in insects (maximal) clutch size is determined early during development in response to larval food conditions ultimately affecting adult size. If this scenario is correct, the trade-off would primarily be a maternal effect that could be achieved by selective abortion of some and reallocation of the energy saved to other eggs, whereas if the trade-off is due to space limitations, it should be largely insensitive to maternal food conditions. Here, high broad sense and low narrow sense heritabilities for egg and clutch size indicate that these traits are strongly affected by maternal effects due to energy limitation (plus perhaps dominance and epistatic variance, as common environment variance was removed); that is, they are maternal as opposed to offspring traits (Roff 1997, p. 259). Food consumption rate, utilization efficiency and energy allocation may thus differ in abundant and limited food environments, differentially affecting the trade-off between egg size and number (Kause et al., 1999; Zera and Harshman, 2001). Nevertheless, yellow dung fly females are able to invest considerably in reproduction even at limited food (Jann and Ward, 1999; this study). Once again, this species exhibits a high degree of phenotypic plasticity, in this case probably ultimately in the (physiological) mechanisms affecting energy allocation and utilization, the evolution of which is generally facilitated by their unpredictable larval environment (cf. Blanckenhorn, 1998, 1999). Lastly, clutch size was positively correlated with body size in our study but egg 
volume was not. This is in agreement with many ectotherms, where egg numbers are strongly dependent on body size whereas this is not necessarily the case for egg size (Wootton 1979; Shine 1988; Roff 1992, p.352; Stearns 1992, p. 174; Fox, 1993; Honek 1993).

In conclusion, our field study of larval and our laboratory study of adult life history traits showed that the trade-off (i.e. the phenotypic and genetic correlations) between development time and body size and that between egg size and clutch size were both weak and stronger in the food limited environment. With regard to the question of how environmental stress affects heritabilities, we found the whole range of possible responses within one single species, depending on the traits considered. All three patterns (or hypotheses) outlined in the introduction occurred (increased $h^{2}$ due to increased $V_{\mathrm{G}}$ or decreased $V_{\mathrm{P}^{\prime}}$ decreased $h^{2}$ due to increased $V_{\mathrm{P}^{\prime}}$ and no change in $h^{2}$ due to increased $V_{\mathrm{G}}$ and $\left.V_{\mathrm{P}}\right)$ occurred, and could be explained by the particular ecological circumstances yellow dung flies face in their natural environment. This may well be the case in other organisms, explaining the multiple patterns found (Hoffmann and Merilä, 1999).

\section{Acknowledgements}

This work was supported by a grant from the Swiss National Fund.

\section{References}

Abrams, P.A., Leimar, O., Nylin, S. and Wiklund, C. (1996) The effect of flexible growth rates on optimal sizes and development times in a seasonal environment. Am. Nat. 147, 381-395.

Alatalo, R.V., Gustafsson, L. and Lundberg, A. (1990) Phenotypic selection on heritable size traits: environmental variance and genetic response. Am. Nat. 135, 464-471.

Amano, K. (1983) Studies on the intraspecific competition in dung breeding flies. I. Effects of larval density on the yellow dung fly. Jpn. J. Sanit. Zool. 34, 165-175.

Arnold, S.J. and Wade, M.J. (1984) On the measurement of natural and sexual selection: applications. Evolution 38, 720-734.

Becker, W.A. (1992) Manual of Quantitative Genetics, 5th ed. Students Book Corporation, Pullman, WA.

Bennettova, B. and Fraenkel, G. (1981) What determines the number of ovarioles in a fly ovary? J.Insect Physiol. 27, 403-410.

Blanckenhorn, W.U. (1997) Altitudinal life history variation in the dung flies Scathophaga stercoraria and Sepsis cynipsea. Oecologia 109, 342-352.

Blanckenhorn, W.U. (1998) Adaptive phenotypic plasticity in growth, development and body size in the yellow dung fly. Evolution 52, 1394-1407.

Blanckenhorn, W.U. (1999) Different growth responses to food shortage and temperature in three insect species with similar life histories. Evol. Ecol. 13, 395-409.

Blanckenhorn, W.U. (2002) The consistency of heritability estimates in field and laboratory in the yellow dung fly. Genetica 114, 171-182. 
Carrière, Y. and Roff, D.A. (1995) The evolution of offspring size and number: test of the SmithFretwell model in three species of crickets. Oecologia 102, 389-396.

Cheverud, J. M. (1988) A comparison of genetic and phenotypic correlations. Evolution 42, 958-968.

Crnokrak, P. and Roff, D.A. (1995) Dominance variance: associations with selection and fitness. Heredity 75, 530-540.

Czesak, M.E. and Fox, C.W. (2003) Evolutionary ecology of egg size and number in a seed beetle: genetc trade-off differs between environments. Evolution 57, 1121-1132.

Davidowitz, G., D'Amico, L.J. and Nijhout, H.F. (2004) The effects of environmental variation on a mechanism that regulates insect body size. Evol. Ecol. Res. 6, 49-62.

de Moed, G.H., de Jong, G. and Scharloo, W. (1997) Environmental effects on body size variation in Drosophila melanogaster and its cellular basis. Genet. Res. 70, 35-43.

Ebert, D. (1993) The trade-off between offspring size and number in Daphnia magna: the influence of genetic, environmental and maternal effects. Arch. Hydrobiol. 4, 453-473.

Ebert, D., Yampolsky, L.Y. and Stearns, S.C. (1993) Genetics of life history in Daphnia magna. I. Heritabilities at two food levels. Heredity 70, 335-343.

Falconer, D.S. (1989) Introduction to Quantitative Genetics, 3rd edn. Longman Scientific and Technical, Harlow.

Foster, W. (1967) Hormone-mediated nutritional control of sexual behavior in male dung flies. Science 158, 596-597.

Fox, C.W. (1993) Maternal and genetic influences on egg size and larval performance in a seed beetle (Callosobruchus maculatus) - multigenerational transmission of a maternal effect. Heredity 73, 509-517.

Fry, J.D. (1992) The mixed-model analysis of variance applied to quantitative genetics: biological meaning of the parameters. Evolution 46, 540-550.

Gebhardt-Henrich, S.G. and van Noordwijk, A.J. (1991) Nestling growth in the great tit. I. Heritability estimates under different environmental conditions. J. Evol. Biol. 3, 341-362.

Gibbons, D.S. (1980) Prey consumption, mating, and egg production in Scathophaga species (Dipt., Scathophagidae) in the laboratory. Entomol. Month. Mag. 116, 25-32.

Grill, C.P., Moore, A.J. and Brodie, E.D. III. (1997) The genetics of phenotypic plasticity in a colonizing population of the ladybird beetle, Harmonia axyridis. Heredity 78, 261-269.

Hoffmann, A.A. and Parsons, P.A. (1991) Evolutionary Genetics and Environmental Stress. Oxford University Press.

Hoffmann, A.A. and Merilä, J. (1999) Heritable variation and evolution under favourable and unfavourable conditions. Trends Ecol. Evol. 14, 96-101.

Honek, A. (1993) Intraspecific variation in body size and fecundity in insects: a general relationship. Oikos 66, 483-492.

Jann, P., Blanckenhorn, W.U. and Ward, P.I. (2000) Temporal and microspatial variation in the intensities of natural and sexual selection in the yellow dung fly Scathophaga stercoraria. J. Evol. Biol. 13, 927-938.

Jann, P. and Ward, P.I. (1999) Maternal effects and their consequences for offspring fitness in the yellow dung fly. Funct. Ecol. 13, 51-58.

Kause, A., Saloniemi, I., Haukioja, E. and Hanhimaki, S. (1999) How to become large quickly: quantitative genetics of growth and foraging in a flush feeding lepidopteran larva. J. Evol. Biol. 12, 471-482.

Kozlowski, J. (1992) Optimal allocation of resources to growth and reproduction: implications for age and size at maturity. Trends Ecol. Evol. 7, 15-19.

Lande, R. and Arnold, S.J. (1983) The measurement of selection on correlated characters. Evolution 37, 1210-1226.

Lazarevic, J., Peric-Mataruga, V., Ivanovic, J. and Andjelkovic, M. (1998) Host-plant effects on the genetic variation and correlations in the individual performance of the Gypsy Moth. Funct. Ecol. 12, 141-148.

Lessells, C.M., Cooke, F. and Rockwell, R.F. (1989) Is there a trade-off between egg weight and clutch size in wild lesser snow geese, Anser caerulescens caerulescens? J. Evol. Biol. 2, 457-472. 
Lynch, M. and Walsh, B. (1998) Genetics and Analysis of Quantitiative Traits. Sinauer Associates, Sunderland, Mass.

Mappes, T. and Koskela, E. (2004) Genetic basis of the trade-off between offspring number and quality in the bank vole. Evolution 58, 645-650.

Merilä, J. (1997) Expression of genetic variation in body size in the collared flycatcher under different environmental conditions. Evolution 51, 526-536.

Møller, A.P. and Swaddle, J.P. (1997) Asymmetry, Developmental Stability, and Evolution. Oxford University Press, Oxford.

Mousseau, T.A. and Roff, D.A. (1987) Natural selection and the heritability of fitness components. Heredity 59, 181-198.

Ojanen, M., Orell, M. and Vaisanen, R.A. (1979) Role of heredity in egg size variation in the great tit, Parus major, and the pied flycatcher, Ficedula hypoleuca. Ornis Scand. 10, 22-28.

Price, T. and Langen, T. (1992) Evolution of correlated characters. Trends Ecol. Evol. 7, 307-310.

Roff, D.A. (1992) The Evolution of Life Histories: Theory and Analysis. Chapman and Hall, New York.

Roff, D.A. (1995) The estimation of genetic correlations from phenotypic correlations: a test of Cheverud's conjecture. Heredity 74, 481-490.

Roff, D.A. (1996) The evolution of genetic correlations: an analysis of patterns. Evolution 50, 1392 1403.

Roff, D.A. (1997) Evolutionary Quantitative Genetics. Chapman and Hall, New York.

Roff, D.A. (2000) Trade-offs between growth and reproduction: an analysis of the quantitative genetic evidence. J. Evol. Biol. 13, 434-445.

Rowe, L. and Houle, D. (1996) The lek paradox and the capture of genetic variance by condition dependent traits. Proc. R. Soc. Lond. B 263, 1415-1421.

Schluter, D., Price, T.D. and Rowe, L. (1991) Conflicting selection pressures and life history tradeoffs. Proc. R. Soc. Lond. B 246, 11-17.

Schwarzkopf, L., Blows, M.W. and Caley, M.J. (1999) Life-history consequences of divergent selection on egg size in Drosophila melanogaster. Am. Nat. 154, 333-340.

Shine, R. (1988) Evolution of large body size in females: a critique of Darwin's fecundity advantage model. Am. Nat. 131, 124-131.

Simons, A. and Roff, D.A. (1994) The effect of environmental variability on the heritabilities of traits of a field cricket. Evolution 48, 1637-1649.

Sinervo, B. and Doughty, P. (1996) Interactive effects of offspring size and timing of reproduction on offspring reproduction: experimental, maternal, and quantitative genetic aspects. Evolution 50, 1314-1327.

Smith, C.C. and Fretwell, S.D. (1974) The optimal balance between size and number of offspring. Am. Nat. 108, 499-506.

Snyder, R.J. (1991) Quantitative genetic analysis of life histories in two freshwater populations of the threespine stickleback. Copeia 1991, 526-529.

Stearns, S.C. (1992) The Evolution of Life Histories. Oxford University Press, Oxford.

$\mathrm{Su}$, G.S., Liljedahl, L.E. and Gall G.A.E. (1997) Genetic and environmental variation of female reproductive traits in rainbow trout. Aquaculture 154, 115-124.

van Noordwijk, A.J. and de Jong, G. (1986) Acquisition and allocation of resources: their influence on variation in life history tactics. Am. Nat. 128, 137-142.

Via, S. (1984) The quantitative genetics of polyphagy in an insect herbivore. II. Genetic correlations in larval performance within and among host plants. Evolution 38, 896-905.

Weigensberg, I. and Roff, D.A. (1996) Natural heritabilities: can they be reliably estimated in the laboratory? Evolution 50, 2149-2157.

Wootton, R.J. (1979) Energy cost of egg production and environmental determinants of fecundity in teleost fishes. Symp. Zool. Soc. Lond. 44, 133-159.

Zera, A.J. and Harshman, L.G. (2001) The physiology of life history trade-offs in animals. Ann. Rev. Ecol. Syst. 32, 95-126. 